\title{
Jung-Quartär und End-Mesolithikum in der Provinz Kerman (Iran)
}

\author{
Von Reinhold Huckriede, Hannover \\ Mit 4 Abbildungen im Text
}

\begin{abstract}
$\mathrm{Z}$ u s a m m e n $\mathrm{f}$ as $s$ ung. Die Hauptmassen der mächtigen jungquartären Schutt-Ausfüllungen und die See-Lösse der weiten Hochtäler des Kerman-Gebietes scheinen unter semi-ariden Bedingungen bis ins ältere Holozän abgelagert worden zu sein. In den heutigen ariden, nahezu wüstenhaften Gebieten nördlich Buhabad und in der Stadt Kerman zeigen Sedimente eines Brackwasser-Sees und süßer bis oligohaliner seichter Gewässer, daß einst Niederschläge und Feuchtigkeit größer waren als heutzutage. In Kerman führen sie mergelig-torfige Lagen und werden dem letzten Pluvial zugeordnet. Sie enthalten viele Farn- und Pilz-Reste und unter den Mollusken feuchtigkeitsbedürftige Landgastropoden, die heute auf dem Iranischen Hochland ausgestorben sind, sich aber in den feuchten Waldgebieten der hyrkanischen Länder finden.
\end{abstract}

Bei Kuhbanan wurde eine mikrolithische Kultur entdeckt, die nach Artefakt-typologischen Gründen als end-mesolithisch zu bezeichnen ist, aber wie das Natufium Palästinas schon viele Sichelklingen mit Cerealien-Kieselglanz aufweist. Damit scheint der Anfang des Getreidebaus in Iran in sehr alten Zeiten oder wenigstens ein Ernten von Wildgräsern und für ihr Gedeihen günstige klimatische Bedingungen bezeugt zu sein.

S u mmary. The main masses of detritus and silts („See-Löß“), deposited in water and filling the vast plateau-valleys of Kerman-area, seem to have been formed during semi-arid conditions till in older Holocene times. North of Buhabad and in the town of Kerman, areas which are now-days arid or nearly desertic, sediments of a brackisch lake and of shallow fresh-or oligohaline waters indicate that once precipitation and humidity were higher than doday. In Kerman they contain layers of marly peat and are thought to be formed during the Last Pluvial Period. They are rich in remains of fungi, ferns, and mollusc, some of which are land gastropods depending on humid environment. These are now extinct on the Iranian Plateau but living in the damp forest areas of the Hyrcanian countries.

At Kuhbanan a microlithic industry was discovered. Regarding the artifact types this industry can be called an Upper Mesolithic. Similar to the Natufian of Palestine there are already plenty sickle blades with a bright patina derived from the silica in the grain stems. This seems to testify the stage of beginning agriculture in Iran in very old times or at least a harvesting of wild grain under climatic conditions suitable for its growth.

\section{Einleitung}

Die Kenntnisse vom Quartär des Iranischen Hochlandes sind noch sehr gering. Einigermaßen gut informiert ist man nur über die heutige und letzteiszeitliche Vergletscherung der Elburs- und Zagros-Gebirgszüge (Zusammenfassung bei Butzer 1958, S. 46, 47), etwas über quartärgeschichtlich auswertbare geländemorphologische Befunde und Sedimente in den Wüstensenken Große Kawir und Lut (Gabriel 1957a, Stratil-SAuer 1957; Bobek 1959a, b) und in Seistan und Nordost-Iran (Huntington 1905, 1907, Scharlau 1958). Eine kurze allgemeine Übersicht über die Sedimente des Quartärs gab Clapp (1940).

Über weite Gebiete der Gebirgsländer sind jedoch kaum quartärgeologische Beobachtungen in der Literatur festgehalten worden. Fast nichts oder nur wenig ist bekannt über quartäre Faunen und Floren, fossile Bodenbildungen und vorneolithische Hinterlassenschaften des Menschen, also über Dinge, die in Iran allein sichere Anhaltspunkte über die Geschichte und die Klimate des Quartärs geben können.

Diese bedauerliche Tatsache ermutigt den Verfasser, einige neben praktischen geologischen Arbeiten für die Bundesanstalt für Bodenforschung in den Hochgebirgen westlich der Lut angefallenen quartär- und steinzeitkundliche Beobachtungen mitzuteilen. Es handelt sich um das Gebiet der großen Hochtäler zwischen Kerman und Buhabad, die zum 
großen Teil nach dem Salz-Endsee von Bafq, zum kleineren in die Lut und die BuhabadWüste entwässern. Eine geologische Karte und Beschreibung des Gebietes wird die Arbeit HuCkriede, Kürsten \& Venzlaff (1961) bringen.

\section{Die heutigen Klima- und Vegetationsverhältnisse}

Nur durch den Vergleich mit dem heutigen Klima und den jetzigen Vegetations- und Faunenverhältnissen, über die BobeK $(1951,1954), R_{\text {ECHINGer }}(1939,1951)$ und Misonne (1959) informieren, lassen sich die quartären Bildungen verstehen. Es sind besonders die sehr geringen Niederschläge, eine lange Trockenperiode im Jahr, beträchtliche jährliche und tägliche Temperatur-Schwankungen, der Steppen- und Wüstencharakter der Vegetation, die das abflußlose Gebiet auszeichnen. Das dem hier behandelten Gebirgsland be-

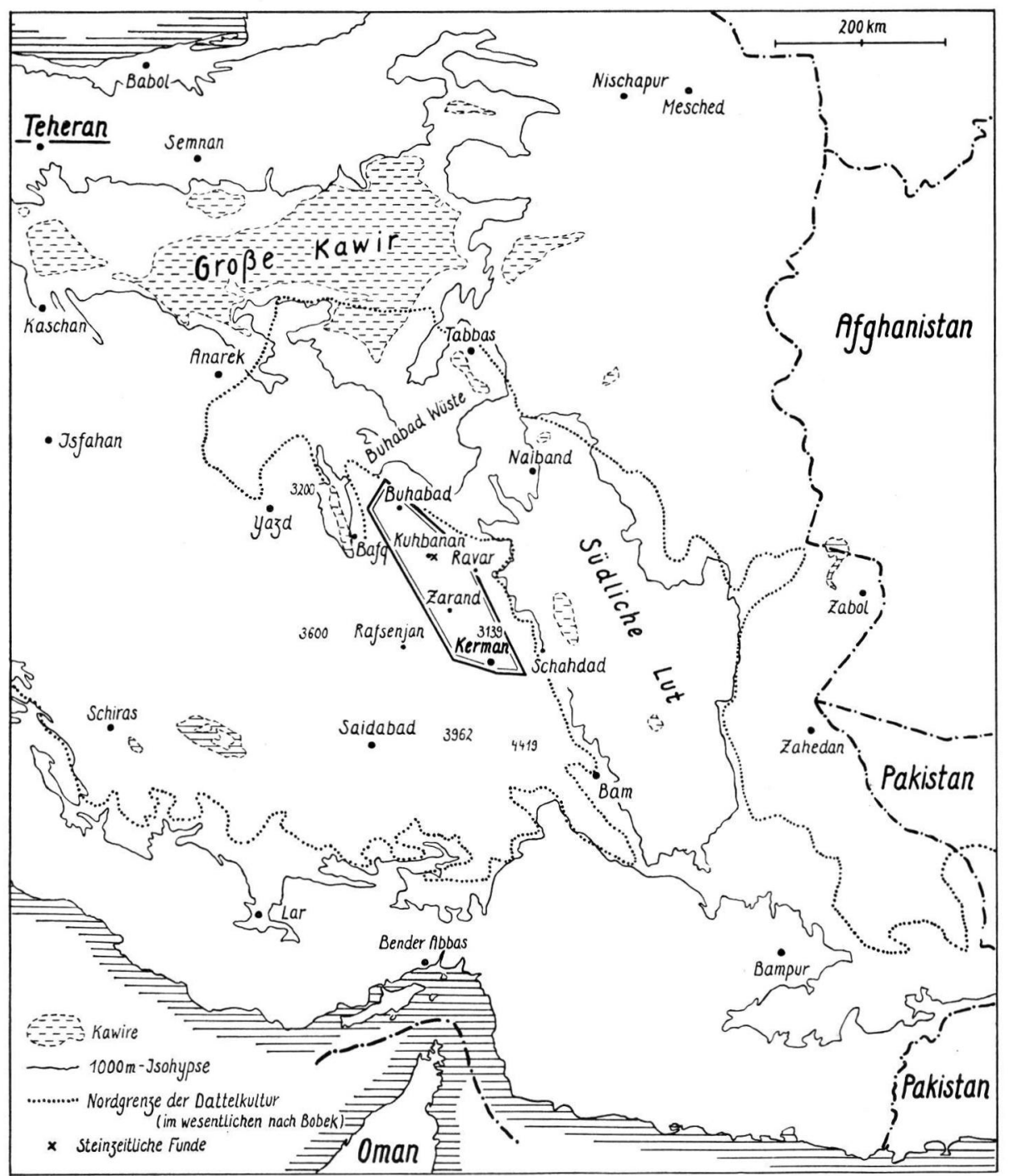

Abb. 1. Schematische Lageskizze des behandelten Gebietes (mit Doppellinie umrandetes Areal). 
nachbarte Becken von Schahdad ist der trockenste Teil der Wüste Lut, Vollwüste mit einem Sandmeer, das bar jeden Lehens und fast völlig ungangbar ist.

Die Gebirge, deren Gipfel gewöhnlich um $3000 \mathrm{~m}$ hoch sind, nahe Kerman fast $4000 \mathrm{~m}$ Meereshöhe erreichen, zeigen stets kahle Berghänge. Nur örtlich sind sie außer mit xerophilen Dornpolstergewächsen schütter mit Bergmandel-Sträuchern (Amygdalus) bewachsen. Der für die Trockengebirge charakteristische Baumwachholder (Juniperus macropoda $=$ excelsa) findet sich nur äußerst selten, und dann nur verkrüppelt, auf für Holzsucher und Kohlenbrenner unzugänglichen Felsen und Geschröf. Als Steppen, Wüstensteppen und Wüsten muß man die weiten Schotter- und Gebirgsschuttflächen, die Senken und breiten Hochtäler bezeichnen. Ausgedehnte Kawir-Regionen findet man nur außerhalb des hier behandelten Gebietes in der Umgebung des Bafqer Endsees und in der Buhabad-Wüste. Größere Flächen der Schwemm-Ebenen sind aber versalzen oder von Sanddünen bedeckt und fast ohne Vegetation; jedoch können hier Saxaule und 'Tamarisken Dickichte bilden.

Die Meereshöhe der hier behandelten Hochtäler liegt zwischen 1400 und $2000 \mathrm{~m}$. Permanente Seen sind nicht vorhanden. Frost und Schneefall sind im Winter verbreitet, mehr als man bei der südlichen geographischen Breite anzunehmen geneigt ist. Wegen des ungünstigen Winterklimas liegt das Gebiet, wie Abb. 1 zeigt, außerhalb der DattelpalmenZone. In Kerman, das $1757 \mathrm{~m}$ hoch liegt, hält sich der Schnee aber nie länger als einige Tage. Auf den außerhalb des behandelten Gebietes liegenden höheren Gebirgsteilen, z. B. dem Kuh-i-Jupar $(3952 \mathrm{~m})$ bleibt der Schnee aber in nordwärtigen Nischen bis in den Juni hinein erhalten. BовEк (1952, S. 78, 79) hat die gegenwärtige ideelle Schneegrenze bei Kerman mit 4900 bis $5000 \mathrm{~m}$ berechnet.

Die mittlere Jahresniederschlagsmenge in Kerman beträgt nach den Angaben von GANJI (1960) und dem Iranischen Wetterdienst für die Zeit von 1950 bis 1959 etwa $170 \mathrm{~mm}$ im Jahr, wobei die Mengen im Laufe der Jahre beträchtlich schwanken (1952= $58 \mathrm{~mm} ; 1957=311 \mathrm{~mm}$ ). Ältere Übersichten (BAUER 1935, Bobek 1952, S. 71) geben nur $105 \mathrm{~mm}$ Durchschnitt an.

In den Gebirgen sind die Niederschlagsbeträge höher, werden hier etwa mit 200 bis $300 \mathrm{~mm}$ (GANJI 1960, Niederschlagskarte) berechnet. In den tieferen Senken werden sie beträchtlich niedriger liegen als in Kerman. Die außerhalb des besprochenen Gebietes, aber noch im gleichen Bafqer Entwässerungssystem liegende Stadt Rafsenjan (1597 m) hat nur $83 \mathrm{~mm}$ Jahresmittel und kennt nach Eвтена (1956) im Jahr durchschnittlich nur 8 Regentage.

Der Regen fällt fast ausschließlich im Winter und Anfang des Frühlings (subtropischer mediterraner Winterregen). Die Zahl der sommerlichen Trockenmonate ohne oder fast ohne Regen liegt bei 6 oder 7 .

Deflation, die in der Lut so eine große Rolle spielt, ist in den behandelten Gebieten nur gering wirksam.

\section{Schwemm-Sedimente semiarider Zeiten; See-Löß}

Die breiten Längstäler zwischen den Gebirgszügen sind mit beträchtlichen Lockermassen ausgefüllt. Nordwestlich Kerman durchteufte eine Wasserbohrung $130 \mathrm{~m}$ dieser Sedimente, ohne ihr Liegendes zu erreichen. An den Rändern der Gebirgszüge, die sich zum Teil als noch aktive tektonische Störungszonen erweisen, sieht man neben Resten von verstellten groben, wohl plio-altpleistozänen Schottern riesige, sich weit in die Ebenen erstreckende, von trockenen Wasserrissen durchfurchte Gebirgsschutt-Fächer und Schlepen. Wo Gebirgstälchen münden, schieben sich of $t$ viele $\mathrm{km}$ lange Wildwasser-Schuttmassen mit grobem Blockwerk in die Hochtäler, Hinterlassenschaften gelegentlicher Wolkenbrüche und Sturzfluten.

In Richtung auf die Innenteile der Senken verkleinert sich das Korn der Schuttmassen, bis schließlich eine tonige Schwemmschluff-Fläche vorliegt, die oft so parketteben und weit 


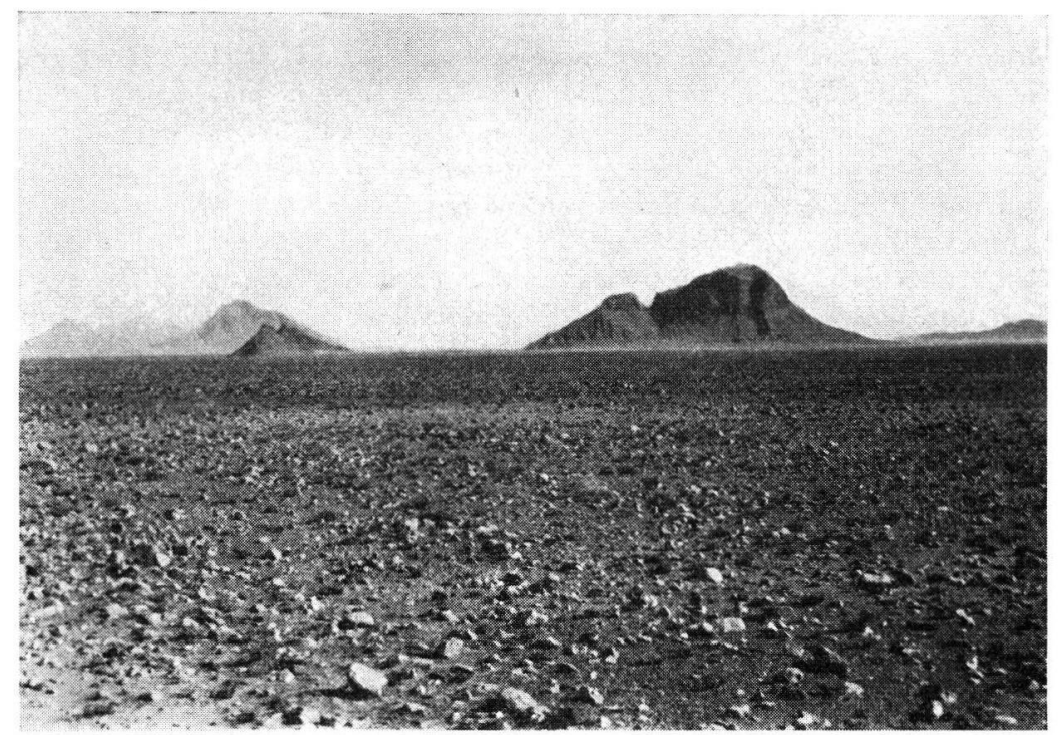

Abb. 2. Blick in das mit Schuttmassen erfüllte Hochtal Kerman-Zarand.

ist, daß Kraftwagen auf ihr wie auf einer Rennstrecke in höchster Geschwindigkeit fahren können. Salzausblühungen sind hier weit verbreitet, und gelegentlich sieht man dünne Gipskrusten.

In dem groben Schuttwerk an den Rändern der Ebenen bieten Wasserrisse vielfach einen Einblick in die oberste, leider immer fossilfreie Sedimentfolge. In den Innenteilen der Becken dagegen, wo man in den feineren Sedimenten Antwort auf stratigraphische Fragen erwartet, fehlen aber meist vertikale Aufschlüsse gänzlich oder gehen nie tiefer als einige Meter. Glücklicherweise bieten die mit den typisch iranischen Grundwassergewinnungsanlagen, den Kanaten, verbundenen Erdarbeiten etwas Ersatz. Sie ergeben bei den Siedlungen viele $\mathrm{m}$ tiefe Einschnitte, oder es werden wenigstens Lockergesteine aus mehreren $10 \mathrm{~m}$ Teufe an die Oberfläche ausgeworfen.

Was man sieht, ist ein unregelmäßig geschichteter Wechsel von tonig-schluffigen Sanden mit unreinem Kies und Grand in Lagen, Schnüren und Linsen und vor allem Pakete etwas sandiger Schluffe, die unter den Bezeichnungen "sekundärer Löß“ (NIEDERMAYER 1920, S. 45), "Seelöß“ (GAbriel 1942, S. 432, S. 308) aber auch als „aeolian silt" (HARRISON, zitiert bei SEDLACEK 1955, S. 623) in die Literatur eingegangen sind.

In den Aufschliissen und im Aushub mangelt es an jedem Zeichen von fossilen Braunerden, roten Bodenbildungen, fossilen Gips- oder Kalkkrusten, fossilen Dünen- oder Wüstensanden. Bis auf die unten von Kerman beschriebenen, wurden auch humose oder torfige Bildungen nicht gefunden.

Die im Aussehen etwas lößartigen, meist gelblichbraunen bis gelblichen, aber auch gelegentlich rot- und violettstichigen kalkhaltigen Schluffe sind gewöhnlich geschichtet und nie so locker wie echter Löß. Wenn sie etwas porös sind, mögen Salzpartikel weggelöst worden sein. Fast nie sind die See-Lösse rein, sondern sie enthalten Sand- und KiesSchmitzen, auch vereinzelt Steinbrocken, was vor allem an der oberflächlichen Windlesedecke erkennbar ist. Häufig finden sich in den oberen Metern auch „Lößkindl“-Konkretionen.

Können die sandigen und kiesigen Lockermassen nach Zusammensetzung und Art der wirren Schichtung nur von periodischen, mit Gebirgsschutt beladenen Wasserfluten herrühren, die sich in vegetationsarme oder wüstenhafte Ebenen ergossen haben, so ist das 
auch der Fall bei den See-Lössen. Diese waren die feinsten Schutt-Teilchen, die Trübe, die sich endlich im zur Ruhe gekommenen Wasser, in flachen, wohl im allgemeinen äußerst kurzlebigen Seen absetzten. Den Absatz im Wasser, nicht eine äolische Entstehung, bestätigt auch die dankenswerter Weise von Dr. SINDowsKI ausgeführte Korngrößen (Pipetten-)Analyse eines solchen See-Lösses von Kerman mit folgenden Werten:

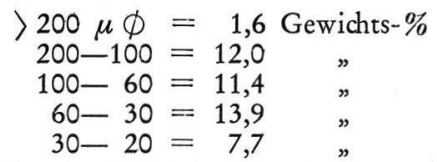

$$
\begin{aligned}
20-10 & =13,2 \text { Gewichts- } \% \\
10-6 & =4,1 \quad " \\
6-2 & =15,8 \quad " \\
2 & =20,8
\end{aligned}
$$

Selbst in den heute staubsturmreichen Gebieten der Lut, wo mit viel mehr äolischem Transport der feinen Teilchen gerechnet werden kann, kam SEDLACEK (1955, S. 618, 619, 623, 656) mit Korngrößen-Analysen zu dem Ergebnis, daß die See-Lösse im Wasser abgelagert worden sind. Ein gewisser Anteil an äolischem Material in der Feinsandfraktion ist hier jedoch wahrscheinlich.

An Schilderungen, wie es zur Bildung von See-Löß, lehmigen, sandigen, kiesigen Schwemm-Massen in den Senken arider oder semiarider Gebicte kommt, mangelt es nicht (z. B. Radde 1898, S. 21; Walther 1900, S. 110; Wenzel 1935, S. 37; Dapples 1941, S. 137; BüDEL 1952, S. 118) $)$ ). Eine gewisse Vorstellung von dem Geschehen geben heutzutage noch die üblichen Winter- und Frühlingsregen im Kermaner Gebiet. Sie breiten feinste Teilchen, den groben Schutt-Bildungen der Ebene selbst entnommen, in den inneren Senken aus. Die seichten Seen, Lachen, Pfützen, in denen das geschieht, werden nach Tagen oder Wochen wieder ausgetrocknet. Dazumal mögen sich aber an manchen Stellen Tümpel von süßem bis oligohalinem Wasser das ganze Jahr hindurch gehalten haben, wofür die Fauna der See-Lösse Hinweise geben könnte.

Ist es nach dem petrographischen Eigenschaften schon klar, daß bei den See-Lössen kein reiner äolischer Löß vorliegt, so beweist auch, wie bei den sehr ähnlichen „fluvioäolischen Ablagerungen" Hadramauts (WENz 1943), die Fauna, daß Wasser bei der Entstehung eine große Rolle spielte. Gewöhnlich sind die Sedimente fossilleer, aber wenn tierische Reste vorhanden sind, ganz gleich aus welcher Teufe, dann sind es

\section{Melanoides tuberciilata (MüLleR) \\ Melanopsis doriae IsseL \\ Pseudamnicola uzielliana (ISSEL)}

In Zarand fand sich einmal Helicopsis (Xeropicta) millepunctata (Boettger).

Alle im See-Löß vorkommenden Mollusken leben noch heute in dem Gebiet, die Helicopsis nur auf den kultivierten Ländereien, die Wasserschnecken in den Bewässerungsund oberirdischen Kanatgräben. Unversalzene größere, permanente Seen, wie man sie sich allgemein für ein Pluvial vorstellt, sind mit den Faunen nicht zu belegen.

Über die Temperaturen, die bei der Sedimentation der molluskenführenden Schluffe herrschten, geben die Melanopsis und Melanoides gute Anhaltspunkte. Es sind wärmebedürftige Arten, die in den aus natürlichen Quellen gespeisten Bergbächen und Wasserspeichern der Gebirge unseres Gebietes nicht gedeihen können, weil ihnen dort das Wasser im Winter zu kalt ist. In den Kanatwasser-Gräben, deren rasch fließendes Wasser stets etwa mit der mittleren Jahrestemperatur aus dem Boden kommt (in Kerman mittlere Jahrestemperatur $17,2^{\circ} \mathrm{C}$ ), setzen die Schnecken in diesem Niveaul aber schon ein, $\mathrm{Me}$ lanopsis doriae allerdings erst mit kleinen Exemplaren. Normale Größen findet man dann in den Gewässern der tieferen Beckenteile. Es mag in diesem Zusammenhang und für die unten behandelten Kermaner limnischen Bildungen erwähnenswert sein, daß Melanoides tuberculata, die "malayische Turmdeckelschnecke“ unserer Warmwasseraquarien, die ja

1) Es sei auch auf die eingehende Behandlung der Pfannen Zentral-Irans durch Gabriel (1957b) hingewiesen. 
eine ungeheure Verbreitung über die warmen Gebiete Afrikas und Asiens hat (siehe Pilsbry \& Bequaert 1927, S. 253, Karte 2; Roger 1943, S. 129), nach den Erfahrungen von KiLIAS (1956) nur in Wassertemperaturen über $18^{\circ}$ vermehrungsfähig ist.

Höchstwahrscheinlich waren die Niederschlagsmengen der Winter- und Frühlingsregen zur Zeit der Ablagerung der Hauptmassen der Hochtal-Füllungen etwas größer als heute. So sind, wie unten gezeigt wird, See-Lösse in Brackwasser-Seen abgelagert worden, wo heute trockenes Land ist. Ferner sprechen die Befunde der Kultur von Kuhbanan für niederschlagsreichere Zeiten im Altholozän und die Feststellung, daß heutzutage in den größten Teilen der Becken die Sedimentation ruht, Abtragung und Akkumulation scheinbar ein Gleichgewichtszustand erreicht haben. Schutthalten liegen seit dem Mesolithikum fossil, wie Artefakt-Funde beweisen, und Wüstensteppen-Flächen mindestens seit Zeiten, wie die Bildung von Wüstenlack auf Geröllen, Dreikantern und Rillensteinen dauert. Zur Zeit der endmesolithischen Kultur von Kuhbanan war dort die See-Löß-Bildung wohl im wesentlichen abgeschlossen. Sie hörte fast gänzlich unter den heutigen ariden Verhältnissen auf.

Keineswegs sind die Lockersedimente aber unter echt humiden Bedingungen und einer üppigen Vegetation abgelagert worden. Nirgendwo fand sich in den jungquartären Serien ein gut sortierter und gut gerundeter Flußkies. Stets wurde grobes Material zusammen mit feinem sedimentiert. Die Gerölle bestehen stets aus Gesteinen, die in den angrenzenden Gebirgsteilen zu finden sind. Nie konnte nachgewiesen werden, daß Gerölle weite Strecken in Längsrichtung der Hochtäler und Gebirgszüge transportiert worden sind. $\mathrm{Zu}$ allen hier behandelten Zeiten wird das Gebiet der Hochtäler keine wasserreichen, immer fließenden und mit unseren europäischen Flüssen vergleichbare Gewässer gekannt haben.

\section{Ablagerungen von Brackwasser-Seen}

Sandige Schluffe, die $25 \mathrm{~km}$ nördlich Buhabad entlang der noch aktiven Störungszone Zarand-Kuhbanan-Buhabad $4 \mathrm{~m}$ tief durch einen jetzt völlig versalzenen, molluskenfreien Bach eingeschnitten worden sind, ergaben

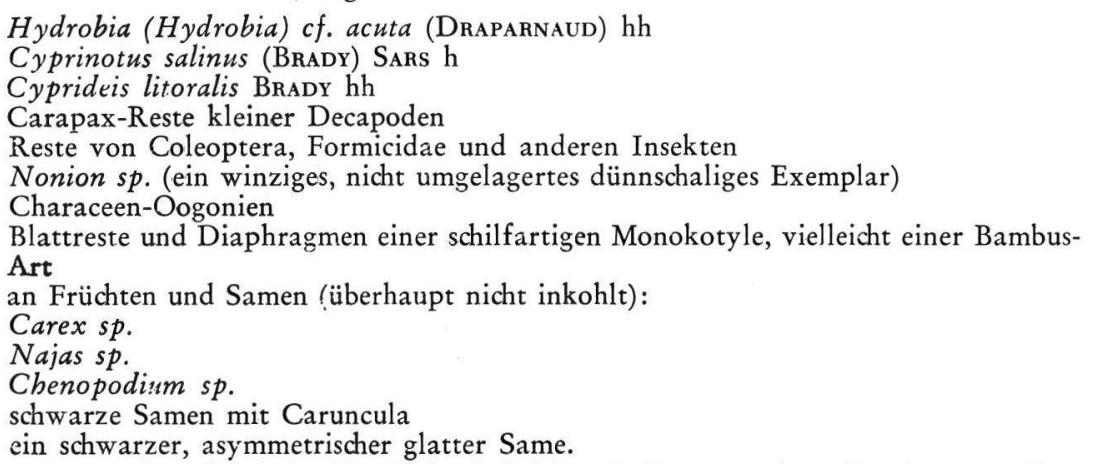

Bei dieser Fauna besteht kein Zweifel, daß hier Sedimente eines Brackwasser-Sees vorliegen. Die auch in der Nord- und Ostsee lebende Brackwasserform Cyprideis litoralis (KuIE 1929, S. 17; 1938, S. 156; Müller 1912, S. 326), die nach Remane \& Schlieper (1958, S. 64, 122) allerdings als holeuryhalin zu bezeichnen ist, konnte der Verfasser lebend auch im Maharlu-Salzsee in Fars nachweisen.

Ein echtes Kawir-Endstadium hat dieser See wohl infolge plötzlicher Hebung des Gebietes, die sich auch in dem scharfen engen Terrassen-Einschnitt anzeigt, nie erreicht. Sollte der See allein das relativ kleine Becken von Buhabad bedeckt haben, würde das bei dem geringen Einzugsgebiet eine sehr niederschlagsreiche Zeit anzeigen.

Es besteht aber die Möglichkeit einer ehemaligen Verbindung zu einem von StratiL- 
SAUER (1957, S. 482) geforderten großen Kawir-See, der sich bis in die Buhabad-Wüste erstreckte. Wertet man die Foraminiferen (Nonion) als Relikte aus einer Zeit der Meeresbedeckung, so muß man einen solchen Kawir-See annehmen und folgern, im Gebiet der Kawir und Lut hätten permante Brackwasser-Seen seit dem Rückzug des Meeres im Neogen existiert, ohne von extremen Trockenzeiten ausgelöscht oder von Pluvialen ausgesüßt worden zu sein. Eine Verschleppung von Nonion durch Seevögel oder anderweitig ist jedoch keineswegs auszuschließen.

Der Begriff Brackwasser soll nur den Salzgehalt angeben und umfaßt im Sinne von REmane (REMANe \& SChlieper 1958, S. 3, 4) auch Binnensalzgewässer, wobei sich selbstverständlich der Verfasser der chemischen Unterschiede zwischen einem mit Meerwasser gemischten Süßwasser und den wohl zumeist Sulfat-Chlorid-Wässern des Iranischen Hochlandes bewußt ist.

\section{Zur Pluvial-Frage}

Unter den Kennern der inneriranischen Trockengebiete, soweit sie sich mit diesen geologischen Fragen in der Literatur auseinandersetzen, scheint nur BoBEK (1955, 1959 a, b) die auf verschiedene Beobachtungen fußende Ansicht zu vertreten, im Iranischen Hochlande hätte es nie eine Pluvialzeit (im stärksten und ursprünglichen Sinne des Wortes) gegeben. Wenn man die geringfügigen gelegentlichen Zunahmen der Feuchtigkeit in der Vergangenheit als Pluviale bezeichnen würde, müsse man es auch mit der Gegenwart tun. Bовек hält z. B. einen großen pleistozänen Kawir-See für unbewiesen, ja glaubt Argumente gegen die Existenz eines solchen für den größten Teil oder gar des ganzen Pleistozäns zu haben.

Die übrigen Forscher sind jedoch von dem Auftreten kräftig niederschlagsreicherer Zeiten in der Vergangenheit überzeugt oder geben Beobachtungen, die man mit reichlichen Niederschlägen zu erklären geneigt ist.

Es sind vor allem geländemorphologische Befunde (Huntington 1905, S. 295-300, Hedin 1910, Niedermayer 1920, S. 53; Gabriel 1957a, Stratil-Sauer 1957, Scharlau 1958), seltener die Untersuchung der Sedimentfolgen selbst (Huntington 1905, 1907, STRATIL-SAUER 1957), die zu solchen, allerdings nicht immer gesicherten oder überzeugenden Schlußfolgerungen führten.

In dem hier behandelten Gebiet spricht mancherlei für ehemals feuchtere und sicherlich auch niederschlagsreichere Zeiten:

Tiefe enge Schluchten mit markanten Strudellöchern sind in den massigen, dichten triadischen Kalk der Gebirgszüge geschnitten, wie es nur ein permanent fließender Gebirgsbach schafft, nicht die schnell erlahmende Wasserflut nach einem der sehr seltenen Sturzregen. Salksinter-Vorkommen, die allerdings nur Steinkerne von Psendamnicola, Pisidium, Characeen-Stengel und Schilf-Röhren geliefert haben, zeugen von ausgedehnteren Quellaustritten dort, wo heute nur ein Quellchen die Bewohner eines kleinen Dorfes am Leben hält oder nicht einmal mehr das vermag.

Auch tiergeographische Hinweise liegen vor, wenn man nicht die Verbreitung der Arten vor das Quartär legt und mit der bei diesen Fällen ziemlich unwahrscheinlichen Verschleppung von Eiern durch Vögel und Wind rechnet.

So kann die vom Wasser abhängige Wechsel- oder Grüne Kröte Bufo viridis viridis LAUR., die unfähig ist, die weiten Wüsten und Wüstensteppen zu überwinden und auf die Oasen und Gebirgsbäche des Gebietes beschränkt ist, sich nur in einer Zeit mit beträchtlich feuchterem Klima über das Iranische Hochland verbreitet haben. Das isolierte Vorkommen von Caspicyclotus biggsi (SCHLESCH) auf schattenbegünstigten Stellen der Hippuritenkalk-Berge bei Kerman (Biggs 1937, S. 347; Schlesch 1934, S. 45) ist nur erklärbar, wenn man eine ursprüngliche Verbindung in feuchteren Zeiten zu dem heutigen Lebensgebiet der Gattung (Bofttger 1889, S. 972, Starmühlner \& Edlauer 1957, S. 445; Forcart 1935, S. 442) im feuchten hyrkanischen Waldgebiet annimmt. Die Ver- 
breitung der Hochgebirgsschnecke Phenacolimax annularis (STUden) (Boettgen 1889, S. 935; BIGGS 1937, S. 343) auf verschiedene, heute durch wüstenartige Senken getrennte Gebirgszüge Zentral-Irans kann auch nur in einer feuchteren Zeit geschehen sein.

\section{Fossile Täler, Terrassenreste}

Nicht selten beobachtet man in den Gebirgszügen Wiederausräumung fossiler Täler oder einst schuttbedeckter Hügellandschaften aus Jura-Gesteinen. Reste alter Schotterfüllungen oder Bedeckung kleben noch hin- und wieder an den Talhängen oder sind als Hügelkappen erhalten. Man könnte aus diesem Wechsel von Schutt-Akkumulation zu Ausräumung einen grundlegenden Wandel der Niederschlagsverhältnisse, des Klimas, könnte Terrassen-Systeme herauslesen und diese auf ein angenommenes, von Europa entlehntes Zeitschema verteilen.

Noch heute bewegen sich jedoch die Gebirgszüge, und die einzelnen Entwässerungssysteme sind somit dem raschen Wandel unterworfen. Entlang aktiven Störungslinien müssen sich Bäche einschneiden, und ganz junge Bewegungslinien ziehen "Terrassen“ in den jungen Schuttfächern der Hochtäler. Angesichts dieser Beobachtungen läßt man lieber die morphologischen Erscheinungen für paläoklimatologische Fragen außer Betracht.

\section{Sedimente von Süßwasser- bis oligohaliner Seen}

Grüne oder grünlichweiße Mergeltone, wie sie in Kanat- und Brunnenausschachtungen in Kerman oder im Bett des Kuhbanan-„Flusses“ unter See-Lössen angeschnitten sind, können nur unter permanenter Wasserbedeckung abgelagert worden sein. Nach HuntingTON (1905, S. 280) bilden Tone solcher Farbe den Boden des Süßwasser-Sees Hamun in Seistan. Bis auf einen Deckel von Melanopsis aus dem höchsten Teil dieser Bildung in Kuhbanan erwiesen sie sich immer als fossileer.

Zum Teil humose, torfige See-Ablagerungen fanden sich in Kerman, als im Zentrum der Stadt auf dem Schulhof der Oberschule ein Brunnen gegraben wurde.

Das Profil:

0- 7,75 m kalkhaltiger Schluff mit etwas beigemengtem Feinsand, gelbbraun, fossilleer, im Oberteil mit Skelett-Resten von Homo sapiens L. (islamischer Friedhof) = endpleistozäner-holozäner See-Löß

7,75- 8,2 m Gemenge weißer Mergel mit schwärzlichen humosen Mergeln

Probe bei $8,0 \mathrm{~m}$ : Pseudamnicola uzielliana (ISSEL) Planorbis sp. indet.

$8,2-9,8 \mathrm{~m}$ weißgraue Mergeltone bis Mergel

9,8 -10,4 m schwarze, torfige Mergel mit

Succinea $s p$. indet.

Vertigo antivertigo Draparnaud

Galba truncatula (MüLleR)

Valvata piscinalis (MülleR), Formen, die sich gut einpassen in Favré's (1927, Taf. 19, Fig. 25-31), "mode minor" und die der V. piscinalis pulchella STUDER nahestehen.

Cyprinotus salinus (Brady) Sars n. ssp. 2

Candona rostrata Brady \& Norman 2

Eucypris pigra (FISCHER) G. W. MüLLER 1

Ilyocypris gibba (RAMDOHR) BRADY n. ssp. („recta“) 6

$10,4-11,9 \cdot m$ (Endteufe): grüne und schmutziggrüne, weißfleckige Mergeltone mit nicht abgerollten Gesteinsbröckchen von $\sim 1 \mathrm{~mm} \varnothing$

Probe bei $11,9 \mathrm{~m}$ : Succinea sp. indet.

Planorbis sp. indet.

verkohlte und zum Teil leicht verkieste Stengel.

Vom torfigen, humosen Auswurf wurden größere Mengen ausgeschlämmt.

Sie ergaben:

Succinea (Succinea) putris h.

Succinea (Succinea) aus dem putris-Kreis, mit verlängertem Gewinde h 
Vertigo antivertigo Drapaknaud $\mathrm{h}$

Planorbis planorbis (L.) (gedeiht noch in Gewässern mit 4-5\% Salz)

Galba truncatula (O. F. MülLER) h (3\% Salz)

Pseudamnicola kotschyi v. Frauenfeld

Valvata piscinalis (MüLLER), der pulchella STUder nahestehend h (2-3\% Salz)

Pisidium cinereum Alder (3\% Salz)

Pflanzen-Stengel

keine Samen und Früchte

Heller Mergel-Aushub ergab nach den Bestimmungen von Dr. Lütrig:

Cyprinotus salinus (Brady) SARS 2

Cyprinotus salinus (Brady) SARS n. ssp. 2

Cyprinotus fretensis (BRADY \& ROBERTSON) 2

Cyprinotus aff. imus GaUTHIER 1

Candonopsis n. sp. 1

Candona aff. vavrai (KaUfmanN) 1

Candona steinheimensis SIEBER 2

Cypridopsis aff. brevisetosa KLIE 2

Ilyocypris gibba (RAMDOHR) BRADY n. ssp. („recta“) 10

Ilyocypris n. sp. 1

Der Unterschied der fossilen zu der heute im Gebiet lebenden Molluskenfauna sticht sofort ins Auge. In dem extrem ariden Klima gedeihen heute nur wenige Formen. Es sind nach eigenen Aufsammlungen und den Angaben bei Issel (1866), Annandale \& Prashad (1919, S. 35), Biggs (1937), Starmühlner \& Edlauer (1957) und Forcart (1959) außer den oben bei den See-Lössen erwähnten sehr häufigen Arten nur noch:

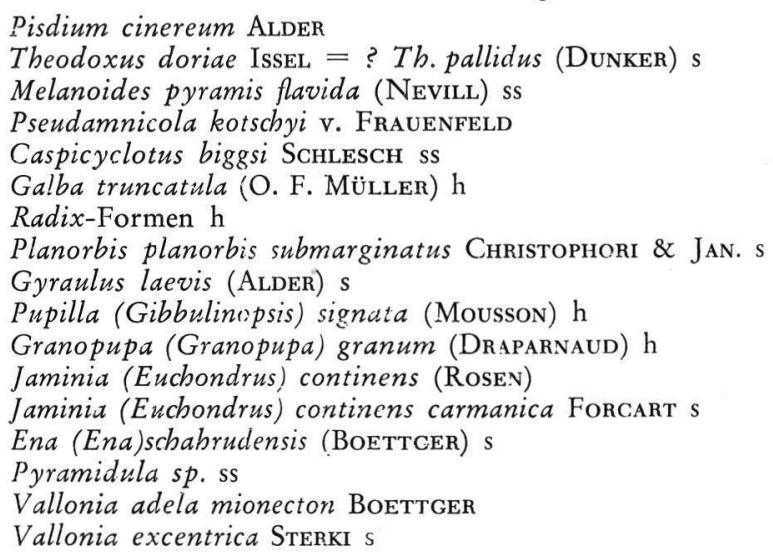

Die jetzt ungeheuer häufigen wärmeliebenden Melanopsis und Melanoides fehlen in der fossilen Kermaner Fauna. Sie müßten nach den aus der Fauna zu schließenden Süßwasser- bis oligohalinen Bedingungen vorhanden sein, wenn die damaligen Temperaturen den heutigen entsprochen hätten.

Valvaten sind noch nie aus Iran beschrieben worden. Der Verfasser suchte sie in dem Gebiet zwischen Fars, Persischem Golf und Seistan vergeblich, und auch der derzeit beste Kenner der iranischen Molluskenwelt Dr. Forcart kennt keine Valvaten von Iran. Valvata piscinalis pulchella STUDER, der unsere Form nahe steht, gedeiht heute nur in Nord- und Mitteleuropa (Ehrmann 1956, S. 207), wird jedoch in Griechenland und Sizilien durch ähnliche Formen vertreten. $V$. pulchella wird auch aus pleistozänen SeenAblagerungen der Türkei genannt (EGERAN \& LAHN 1948).

Es ist undenkbar, daß in Kerman bei den heutigen ariden Verhältnissen mit stetem Ausblühen von Salz nahe den Gewässern feuchtigkeitsliebende Vertigonen und Succineen gedeihen könnten. Vertigo antivertigo ist wohl heute gänzlich auf dem Iranischen Hochland ausgestorben. Man kennt die Art in Iran einzig in den feuchten Waldgebieten nörd- 
lich des Elburs-Kammes, von wo sie Forcart (1935, S. 420), erwähnt, in einem Gebiet, das eine vollkommen andere, ungemein reichhaltigere Landgastropoden-Fauna birgt als das Iranische Hochland. Auch die Succineen werden nur noch an ganz wenigen feuchtig. keitsbegünstigten Orten des Hochlandes leben, wie Succinea pfeifferi Rossm. in dem Auen-Gebüsch des Zeyandeh Rud bei Isfahan (BrgGs 1937, S. 347). Ein wahrscheinlich zu dieser Art zu stellendes Gehäuse erwähnt BIGGs übrigens aus quartären Ablagerungen bei Bam. Iranische Succinea putris sind meines Wissens bislang nur aus dem feuchten Waldland nördlich des Elburs-Kammes bekannt gemacht worden (STARMüHLNER \& EdLAuer 1957, S. 464).

Die Ostracodenfauna von Kerman enthält Formen, die gelegentliches Austrocknen kleinerer Gewässer vertragen. So ist Cyprinotus fretensis eine für kleine Wasseransammlungen typische Form, die in Nordafrika und Bulgarien häufig in Feldpfützen gefunden worden ist. Alle genauer bestimmten Arten sind gegen hohe Wassertemperaturen, wie sie beim Austrocknen entstehen, unempfindlich. Fließgewässer, ausgenommen kleine, nicht perennierende, kommen als Biotop nicht in Betracht. Daß die Wasserfläche nicht allzu klein war, gibt Ilyocypris gibba „recta“ zu erkennen, eine limnische, Seen bevorzugende Form. Auf anmooriges Gewässer deutet Candona rostrata hin. Nach $\mathrm{K}_{\mathrm{LIE}}(1938, \mathrm{~S}$. 40) kommt sie in moorigen Gräben und an seichten Seeufern zwischen dichtem Pflanzenwuchs vor. Die nicht näher bestimmten Arten lassen sich ökologisch nicht auswerten. Doch widersprechen die ökologischen Merkmale der verwandten Arten gleicher Gattung unseren Annahmen nicht.

Einige Arten sind noch nicht beschrieben, was angesichts der ungenügenden Kenntnis von den iranischen Kleinfaunen nicht wunder nimmt.

Candona steinheimensis ist in Deutschland bisher nicht in jüngeren als altpleistozänen Schichten bekannt geworden. Ilyocypris gibba "recta" scheint im Mittelmeer-Gebiet ebenfalls nicht bis in jungpleistozäne Ablagerungen zu gehen. Für die stratigraphische Einordnung der Kermaner Sedimente brauchen diese Feststellungen aber keine Bedeutung zu haben.

Einen ausgedehnten, tiefen See nach mitteleuropäischen Vorstellungen gab es bei Kerman sicherlich nicht. Ein solcher hätte nach den bei den pluvialzeitlichen Seen Kleinasiens gewonnenen Erfahrungen Fischreste, Unioniden, Dreissensien, höchstwahrscheinlich auch Corbicula fluminalis überliefert. Die Ebene von Kerman wird vielmehr nur von einem ganz seichten See, vielleicht auch nur von Tümpeln und Lachen bedeckt gewesen sein, von denen große Teile immer wieder trocken fielen und von Landpflanzen überwuchert wurden.

Aus dem Vorkommen von Cyprinotus salinus könnte auf eine leichte Salzführung des Wassers geschlossen werden.Auch die in der Fundliste mit Grenzwerten des Salzgehaltes (nach Remane \& SCHLIEPER 1958, S. 77, 78) versehenen Mollusken gedeihen in oligohalinem Wasser. Besonders die Psendimnicola-Arten vertragen viel Salz (STARmühlner \& Edlauer 1957, S. 444). Alle diese Arten sind aber auch gute Süßwasser-Bewohner, selbst C. salinus wird aus reinem Süßwasser aufgeführt (VAvra 1909, S. 107).

Eine leichte Versalzung des Wassers ist aber wahrscheinlich. Selbst eine niederschlagsreichere Zeit, ein Pluvial, wird in diesen abflußlosen Gebieten die aus den ariden Zeiten überkommene Versalzung der Wässer und Böden nicht aufheben können. Zudem enthält das Gebiet in allen Formationen vom tiefsten Kambrium an, vor allem im Neogen, die mannigfaltigsten salinaren Gesteine, so daß selbst in einem sehr humiden Pluvial mit Quellwässern geringen Salzgehaltes zu rechnen wäre.

Die Hoffnungen, die auf eine pollenanalytische Untersuchung der torfigen Schichten von Kerman gesetzt wurden, wurden leider enttäuscht. Oxydation hat wahrscheinlich alle Pollen zerstört, denn solche ließen sich nicht nachweisen. 
Herr MädLen fand in der Probe aus $8 \mathrm{~m}$ Tiefe:

kleine Holzsplitter, teils von Farnen

Pilzsporen

Algen (Pediastrum; 5\% Salz).

Die Schicht 9,8-10,4 m ergab:

kleine Holzsplitter, teils von Farnen, aber in größerer Anzahl als oben, einfache Pilzsporen

Teleutosporen

Pilz-Hyphen

Pseudopasenchym von Pilzkörpern

Kutikulae mit stark verzahnten, langgestreckten Zellen, wie sie bei Gräsern vorkommen.

Man erkennt also, daß reichlich pflanzliches Material anfiel, die Vegetation nicht so arm und dürftig war wie heutzutage unter natürlichen Verhältnissen bei Kerman. Bemerkenswert ist das Fehlen von Früchten und Samen größerer Sträucher und Bäume und die Häufigkeit von Farn-Resten. Vielleicht bestand die Vegetation vorherrschend aus Farnen, und weite Gebiete waren mit Farn-Heiden bedeckt. Es ist ja bekannt, daß der Adlerfarn Pteridium aquilinum in afrikanischen Gebirgen eine ganze Pflanzenformation bildet und im Himalaya bis $2500 \mathrm{~m}$ hinaufgeht. BoBEK (1951, S. 43) erwähnt in den feuchten kaspischen Wald-Regionen Adlerfarn-Heiden, die gern die erschöptten Kulturböden überwuchern und schlecht wieder auszurotten sind.

Adlerfarne gibt es aber heute nicht mehr in der Provinz Kerman. Überhaupt hat der Verfasser während seiner achtmonatigen Kartierzeit und weiten Fahrten hier nirgendwo Farne gesehen, mit Ausnahme einiger seltenen zierlichen Asplenium in dämmerigen, feuchten Kanat-Stollen.

$\mathrm{Ob}$ nun zur Zeit des Kermaner-Sees bei Kerman Auewälder oder selbst in den Gebirgen Wälder gediehen, die feuchter als die trockenen Bergwacholder-Wälder oder gar feuchter als die an Unterholz mangelnden schütteren Eichenwälder des Zagros waren, kann man aus den Befunden nicht erschließen.

Ein feuchter Wald von der Art des Kaspischen Bergwaldes (vgl. BoBEK 1951, S. 18), wie er heute an der Nordseite des Elburs die Höhen von etwa $1000-2000 \mathrm{~m}$ mit seinen Buchen, Eichen, Weißbuchen, Eiben, Stechpalmen und einem reichen Unterwuchs von Kräutern, Farnen, Pilzen auf braunem Waldboden bedeckt, müßte doch in den Quartärprofilen sich hie und da mit einer typischen Bodenbildung kundtun. Aue- und TalgrundDickichte aus Erlen, Ahornen und Weiden liegen für die Zeit des Kermaner Sees aber nicht außerhalb der Möglichkeit.

Es sei auch daran erinnert, daß die theoretische Forschung während des letzten Pluvials einen gemäßigten bzw. subtropischen Wald entlang der westlichen iranischen Gebirge weit nach SE vorgedrungen annimmt (Butzer 1958, S. 140 „Tentative Sketch of Würm Climatic Vegetational Zones").

Wenn man jedoch RECHINGER's „Grundzüge der Pflanzenverbreitung im Iran“ liest, dann erfährt man, welche reicher Endemismus an Gattungen und Arten auf dem Iranischen Hochland herrscht, daß z. B. eine Gipswüste bei Semnan unter weniger als 20 Pflanzenarten fast zwei Drittel Endemiten und zwar fast durchaus Arten von isolierter systematischer Stellung, beherbergt. So ist es wohl klar, daß für ausgedehnte Areale, besonders der tieferen Lagen des Hochlandes, auch die Zeiten der eurasiatischen Glaziale keine wesentlichen Veränderungen der Klima- und Vegetationsbedingungen gebracht haben können, oder extreme Xerophyten immer Ausweichmöglichkeiten in benachbarten Gebieten gehabt haben müssen.

Fassen wir zusammen, was bei den humosen Schichten Kermans für ein etwas kühleres, feuchteres Klima - höchstwahrscheinlich für ein echtes, wenn auch nicht zu kräftiges Pluvial - spricht: 
1. mindestens $3 \mathrm{~m}$ mächtige Ablagerungen eines seichten Süßwasser- bis oligohalinen Sees dort, wo heute unter natürlichen Bedingungen Wüstensteppen-Verhältnisse herrschen und der Grundwasserspiegel 10 bis $12 \mathrm{~m}$ tief liegt.

2. feuchtigkeitsabhängige Landgastropoden, die heute in Iran nur in den feuchten Waldgebieten Hyrkaniens oder nur an ganz wenigen Feuchtigkeit-begünstigten Orten des Hochlandes gedeihen.

3. „nord- und mitteleuropäische“ Valvaten, die heute in ganz Iran ausgestorben zu sein scheinen.

4. Fehlen der wärmebedürftigen, heutzutage überaus zahlreichen Melanopsis und Melanoides in den Ablagerungen.

5. Anreicherung von Vegetationsresten zu torfigen Lagen, dort wo heute auf versalzenen Wüstensteppenböden nur dürftige xerophile Kräuter gedeihen.

6. Neben reichlicher Pilz-Flora häufige Farne, die heutzutage im Gebiet nicht mehr $\mathrm{zu}$ finden sind.

Was das Alter dieser feuchtzeitlichen Bildung anbelangt, so wird man diese wohl nur in das wahrscheinlich in die Periode der Würm-Vereisung fallende Letzte Pluvial stellen können.

\section{Fehlen von Höhlensedimenten und glaziären Bildungen}

Obgleich mächtige Hippuriten-, Rudisten- und Apricardien-Kalke im ganzen Gebiet weit verbreitet sind, findet man an der Oberfläche keine Verkarstungs-Erscheinungen und Höhlen. Das mag an der raschen Hebung der Gebirge und an den geringen Niederschlägen liegen. Mit höhlenkundlich-stratigraphischen Ergebniisen ist also in diesem $\mathrm{Ge}-$ biet kaum zu rechnen.

Gänzlich fehlen in den Gebirgen des hier besprochenen Gebietes glaziär-morphologische Formen oder Moränen. Die sehr hohen Gebirge südöstlich und südlich Kerman wurden allerdings nicht auf Vergletscherungs-Spuren untersucht.

\section{Die end-mesolithische oder protoneolithische Kultur von Kuhbanan}

Nahe einer Mausoleums-Ruine und einem alten islamischen Friedhof am östlichen Ortsrand von Kuhbanan, dem Kobinam bzw. Cobinan MARco Polo's, stieß der Verfasser auf eine bisher unbekannte Steinzeit-Station. Zahlreiche mikrolithische Artefakte lagen hier auf einer leicht mit Hangschutt bedeckten See-Löß-Terrasse von etwa $4 \mathrm{~m}$ Höhe.

Es wäre zu begrüßen, wenn dieser Platz von Fachleuten untersucht werden würde, da ja zwischen den bekannten mesolithischen bzw. protoneolithischen Kulturen Syriens und des Iraks und den Fundplätzen von Mikrolithen in Pakistan und Indien (siehe WheELER 1959, Abb. 14) in Iran eine große Fundlücke klafft, nach der Übersicht von VANDEN BERGHE (1954) im ganzen Iranischen Hochland von Kaschan an bis Belutschistan und Seistan überhaupt noch keine Steinzeit-Funde gemeldet worden sind.

Auch sonst ist in Iran nur wenig von Kulturen bekannt, die älter als jene von Tepe Sialk I (GHIRSHMAN 1938; 1954, S. 29-32) und älter als echtes Früh-Neolithikum sind. Es sind, abgesehen von den Moustérien-Oberflächenfunden von Khunik in Khorasan (Coon 1951) stets Funde in und bei Höhlen, die schnell aufgeführt sind:

In Mazanderan: Ghar-i-Kamarband mit Spät-Mesolithikum und vorkeramischem Neolithikum; Ghar-i-Hotu mit Mittel- und Jungpaläolithikum, Mesolithikum (Coon 1951; 1952; Vanden Berghe 1954, S. 350-352; Gross 1957, S. 158)

In Luristan: Tang-i-Pabda mit Altneolithikum (Ghirshman 1954, S. 27, 28; Braidwood 1953, S. 307) 
In Aserbaidschan: Tamtama mit ? Levalloisien (Coon 1951, VANDEN Berghe 1954, S. 387)

In Kurdistan: "Hunters cave" bei Bisutun = Behistun mit jungem Levalloiso-Moustérien und ? Mesolithikum (Coon 1951, Movius 1951).

Wenn man einer Journalisten-Meldung (Linehan 1961, S. 82) Glauben schenken darf, wurde jüngst auch junges Altpaläolithikum in der Gegend von Kermanschah gefunden.

Bei Kuhbanan vermißt man an dem Fundplatz Siedlungs- d. h. Lehmbauten-Schutt, Keramik, Steingefäße, geschliffenes Steingerät, Reib- und Mahlsteine sowie Metallgegenstände, die irgendwie mit dem Geräte-Inventar in Verbindung stehen könnten. Es besteht kein Anlaß, daran zu zweifeln, daß die aufgesammelten Artefakte einer einzigen Kultur zugehören. Abgesehen von einigen mikrolithischen Artefakten bei einer Quelle $6 \mathrm{~km}$ SSE von Kuhbanan (genaue Lageangabe der Fundplätze bei HuCKRIEDE, Kürsten \& VenzlafF 1961), die sicherlich der gleichen Kultur angehören, ist es der einzige Fundplatz von Steinzeit-Artefakten, der während der Kartierzeit in der Provinz Kerman aufgefunden werden konnte. Wenn man sich an die Dichte von mesolithischen Schlag- und Siedlungsstellen erinnert, wie sie manche Gebiete Norddeutschlands aufweisen oder weiß, wie schnell man in den alten Kultur-Gebieten von Fars Stein-Artefakte finden kann, so kann man sich des Eindrucks nicht erwehren, im Alt-Holozän und bis ins Chalkolithikum sei die aride Provinz Kerman nur äußerst dünn vom Menschen besiedelt gewesen.

Das Geräte-Inventar der Kuhbananer Kultur (Abb. 3 und 4) enthält am häufigsten kleine dünne Klingen bzw. Sichelklingen. Besonders schmale Stücke sind nicht einmal $5 \mathrm{~mm}$ breit. Nicht selten sind sie an einer oder an beiden Schneiden zierlich retuschiert (Fig. 2, 4). Gröber retuschierte, dickere Klingen (Fig. 18) und Klingen mit Hohlkehlen Fig. 3) sind seltener.

Prachtvolle pyramidale Kernsteine (Fig. 23-27) sind sehr klein, manchmal nicht höher als 25 oder gar $19 \mathrm{~mm}$. Wahrscheinlich ist es eine Auswahl, die als Geräte Verwendung fand. So sind ihre Spitzen zu Stichel umgearbeitet worden (Fig. 26) oder enthielten eine Querschneide (Fig. 27). Kernsteine sind auch in der Absicht, einen winzigen Druckstein mit Spitze zu erhalten, zu der Kleinheit abgespant und im unteren Teil für Schäftung hergerichtet worden. Ebenfalls fanden Kerne als Hoch-Schaber Verwendung.

Es fand sich auch eine Klinge mit bogenförmig abgedrücktem Rücken (Fig. 13), die an ähnliche im Natufium Palästinas (Garrod 1932, Abb. B, C; Turville-Petre 1932, Abb. F) und im Capsien Nordafrikas (VAufrey 1933, Abb. 2 und 17) erinnert, ferner ein Halbmond-Messer (Fig. 21).

Häufig sind geometrische Mikrolithen in Form von Trapezen (Fig. 48-54, 56), Dreiecken (Fig. 45-47, 55), Vierecken (Fig 57) und Halbmonden (Fig. 31-42) sowie TrapezMesserchen (Fig. 43, 44).

Schaber sind nicht selten. Es handelt sich um Rundschaber bzw. Daumennagel-Schaber (Fig. 19), Halbrund-Schaber (Fig. 20) und (selten) Klingen-Schaber mit einer Randretusche (Fig. 30).

Kleine Spitzen sind von der Art wie jene der Fig. 9-11 und 29, zum Teil jener von NEuville (1934, Abb. 2, Fig. 1) aus dem Natufium III abgebildeten ähnlich. Es liegen auch Bohrer mit feinster Spitze (Fig. 5-7) vor.

Was Mikrostichel bzw. Reststïcke von der Herstellung geometrischer Mikrolithen (vgl. Adrian 1956, S. 23, 24) anbelangt (Fig. 59-61), so sind sie, verglichen mit den Mengen von Dreiecken, Trapezen und Halbmonden, sehr selten. Sie stimmen völlig überein mit jenen des Tardenoisien Frankreichs (z. B. Daniel 1954, Abb. 3, Fig. 32-35; Furon 1958, Abb. 85, Fig. 18-23, 27) und des mitteleuropäischen Mesolithikums (z. B. Reuter 1955, Abb. 6, Fig. 1-4). 

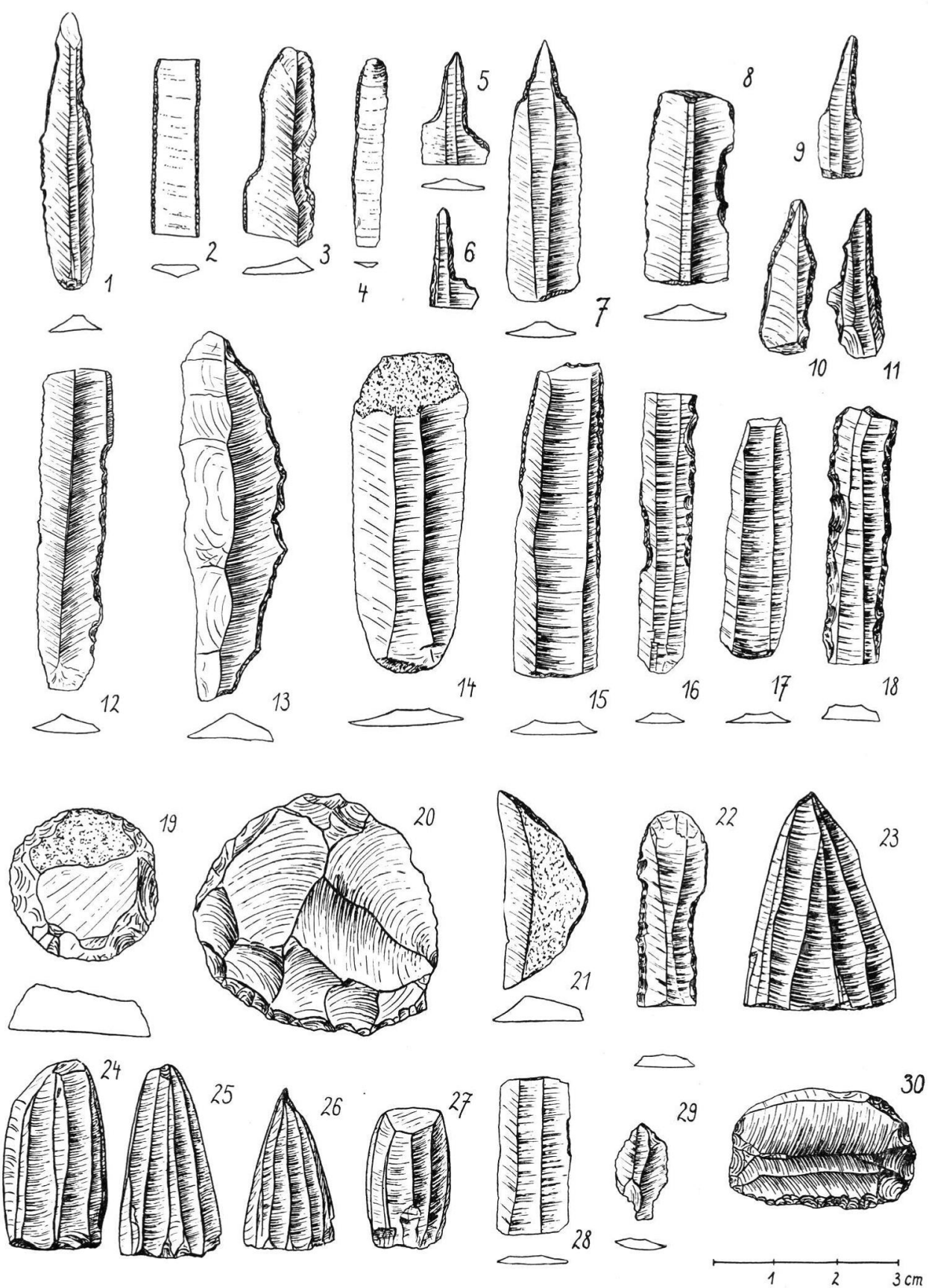

Abb. 3. Gerät-Typen der Kultur von Kuhbanan.

Nicht gefunden wurden in dem umfangreichen Fundgut Eckstichel, Stielspitzen, geometrische Dreieckspitzen und Kernbeile.

Der Werkstoff der Geräte besteht hauptsächlich aus Feuerstein, der gelegentlich Orbitolina und Dictyoconus führt und in der Oberkreide der Umgebung und in den neogenen und quartären 


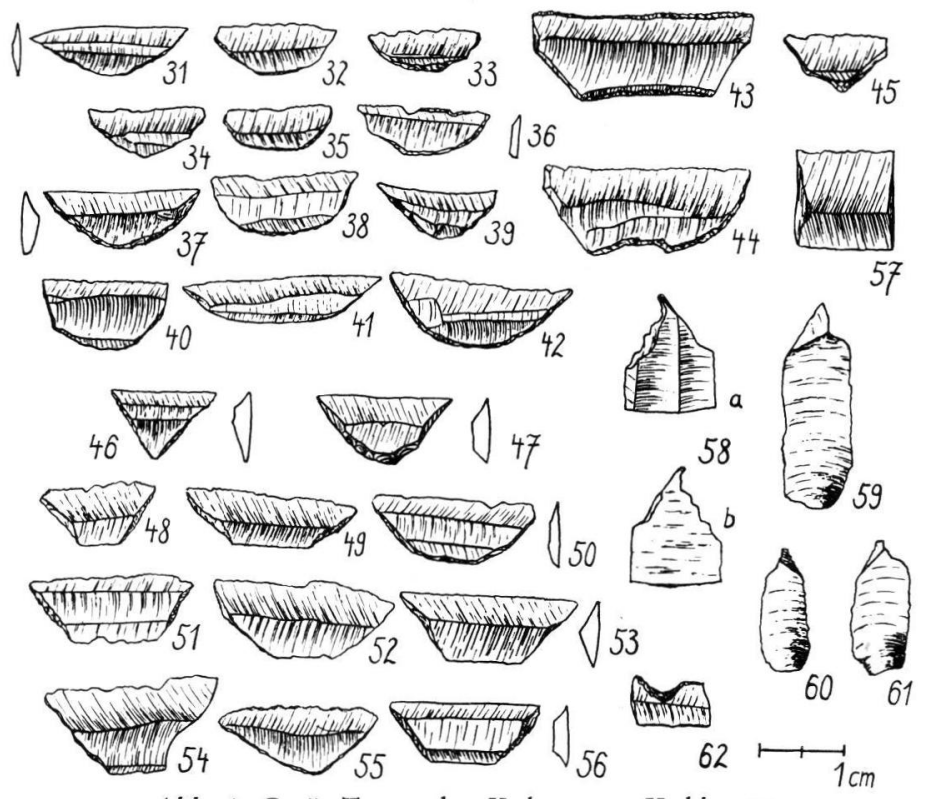

Abb. 4. Gerät-Typen der Kultur von Kuhbanan.

Schottern als Gerölle weit verbreitet ist. Seine Farbe ist gelbbraun bis schokoladenbraun, braunrot, fleischfarben oder weißlich.

Häufig verwendet ist auch ein brauner gebänderter Jaspis, der in dem Gebiet zwischen Bafq und Kerman nicht einheimisch zu sein scheint. Genau so fremdartig sind Obsidian, hellgrüne Radiolarite und dunkle kieselige Schiefer sowie manche Chalzedone. Diese Gesteine kommen seltener vor, zeigen aber, daß die Gerät-Hersteller entweder weite Streifzüge in Iran unternommen haben oder - was weniger wahrscheinlich ist -.. ein Handel mit begehrtem Stein-Rohstoff bestand. Die nächsten in der Literatur (Askıund 1927, S. 416, 418) erwähnten Obsidian-Vorkommen liegen zwischen Tabas und Naiband (etwa $200 \mathrm{~km}$ Luftlinie von Kuhbanan entfernt) und südlich der Großen Kawir nördlich Khur.

Mit dieser Aufzählung sind noch nicht alle verwendeten Gesteinsarten erschöpft. Es wurden noch schwarzer algonkischer Lydit der Morad-Serie (Huckriede, Kürsten \& Venzlaff 1961), weißliche bis rötliche Hornsteine des tiefsten Kambriums und rote, ge!be, braune oder mehrfarbige Eisenkiesel aus den Kontakthöfen der Granite und Granitporphyre, wie sie sich auch z. B. in der Wüste von Bafq als Gerölle finden, Bergkristall und Quarz verwendet.

$\mathrm{Daß}$ Ackerbau getrieben oder wenigstens Wildgräser geerntet wurden, beweist der Cerealien-Kieselglanz auf manchen Klingen. Ein reichhaltiges geometrisches MikrolithInventar, Mikrostichel, Halbmondmesser und Messer mit abgedrücktem Rücken weisen nach typologischen Gesichtspunkten die Kultur als mesolithisch aus. Da ferner Steinschliff, Keramik, Metall und Lehmbauten zu fehlen scheinen, kann man echtes Neolithikum oder gar Chalkolithikum ausschließen. Man hat also mit der Möglichkeit zu rechnen, daß genau so wie in Palästina (Natufium und in Alt-Jericho) oder im Irak (Karim Shahir; Qualat Jarmo) auch auf dem Iranischen Hochland entweder ein präkeramisches Protoneolithikum mit Ackerbau oder ein Übergang vom Sammler-Stadium zum Pflanzenbau oder wenigstens zum Ernten von Wildgras-Samen nachzuweisen sein wird.

Für die Geologie hat die Kuhbananer Kultur folgende Bedeutung:

Die Lage der Artefakte auf einer See-Löß-Terrasse, auf der eine Schutthalde vom Berghang ausklingt, und in der See-Löß-Niederung von Kuhbanan selbst, zeigt, daß die heutigen morphologischen Verhältnisse schon damals bestanden und die holozäne SeeLöß-Bildung zur Zeit der Kuhbanan-Kultur im wesentlichen abgeschlossen gewesen sein muß. Die Artefakte fanden sich - ganz auffällig - vor allem nahe einem jetzt trocke- 
nen Bacheinriß. So wird man zu der Annahme gezwungen, dieser hätte damals noch einen permanent fließenden Bach beherbergt und den Menschen das zum Leben notwendige $\mathrm{Naß}$ gespendet. Sicherlich war die Zeit noch durch größere Niederschlagsmengen als heutzutage begünstigt. Ohne sie wäre auch kein Getreideanbau oder Gedeihen von Wildgräsern denkbar; denn die Kunst der Kanat-Herstellung wird man den Menschen an der Wende Meso-/Neolithikum wohl noch nicht zutrauen können, und heutzutage hängt der Getreidebau, überhaupt das ganze menschliche Leben in Kuhbanan, allein von der Grundwasser-Gewinnung ab.

Am Ende bleibt noch die angenehme Pflicht, meinen Kollegen Dr. VenzLafF und Dr. Kürsten (Bundesanstalt für Bodenforschung) für viele Hinweise aus ihrem Erfahrungsschatz zu danken, ferner den Hetren Davoonzadeh, Vossoughzader und Movahed vom Kaiserlich Iranischen Ministerium für Industrie und Bergbau, die mir bei den Gelände-Arbeiten zur Seite standen und Herrn Ingenieur R. AzARINE, der mich auf das Pluvial von Kerman aufmerksam machte. Großen Dank schulde ich für die Bestimmung der Ostrakoden und ökologische Hinweise Dr. Lürtig, für paläobotanische Untersuchungen Herrn MäDLER, für die Korngrößen-Analyse Dr. Sindowski (alle Niedersächsisches Geolog. Landesamt), für malakozoologische Hilfe Dr. ForCART (Naturhistorisches Museum Basel).

\section{Nachtrag während der Drucklegung}

Eine im Cl4-Labor des Niedersächsischen Landesamtes für Bodenforschung ausgeführte Altersbestimmung ergab für das torfige Sediment von Kerman (9,8-10,4 m Teufe) einen an der Reichweitengrenze der Apparatur gemessenen Wert von $>25000$ Jahren. Er schließt also ein holozänes oder spätglaziales und wahrscheinlich auch ein dem Kältemaximum des Jung-Würm entsprechendes Alter der Schicht aus. Den Herren Dr. WENDT und Dr. Homilius sei vielmals für die Bestimmung gedankt.

Herr Dr. A. RutrneR, Geologische Bundesanstalt Wien, machte mich liebenswürdigerweise auf die übersehene wichtige hydrobiologische Arbeit

LöFFLER, H.: Beiträge zur Kenntnis der iranischen Binnengewässer I: Der Niriz-See und sein Einzugsgebiet. - Internat. Revue d. gesamten Hydrobiologie 44, S. 227-276, 26 Abb., Berlin 1959,

aufmerksam. Neben lebenden Foraminiferen wird auch Cyprideis litoralis im Niriz-See aufgeführt. Wichtig für unsere Betrachtungen ist die Meldung, daß inzwischen Valvata piscinalis pulchella Studer im Zagros-Gebirge und somit zum ersten Male in Vorderasien gefunden worden ist. Sie lebt in Quellbächen bei Asupas (ohne Melanoides und Melanopsis!) in Höhen von 2000 m oder mehr. Die Niederschläge liegen in diesem bis über $4000 \mathrm{~m}$ ansteigenden Teil des Zagros-Gebirges bei über 400 und über $500 \mathrm{~mm}$ im Jahr. Die klimatologischen Aussagen dieser Reliktenposten von Valvata im Zagros stehen somit in gutem Einklang mit den Vorstellungen über das Kermaner pluvialzeitliche Klima.

\section{Angeführte Schriften}

Adrian, W.: Beiträge zur Steinzeitforschung in Ostwestfalen. Teil II. - Ber. Naturwiss. Ver. Bielefeld u. Umgegend, 14, S. 5-12.1, 76 Abb., 1 Karte, 2 Tab., Bielefeld 1956.

Annandale, N. \& Prashad, B.: The Mollusca of the Inland Waters of Baluchistan and of Seistan. - Rec. Indian Mus., 18, S. 17-62, Taf. 3-8, 9 Abb., Calcutta 1919.

AskuUnd, B.: Zur Geologie Ostpersiens auf Grund der von Sven Hedin gesammelten Gesteinsproben. - In Sven Hedin: Eine Routenaufnahme durch Ostpersien, 2, S. 381-446, 17 Taf., Leipzig u. Stockholm (Generalstabens Litografiska Anstalt) 1927.

BAUER, G.: Luftzirkulation und Niederschlagsverhältnisse in Vorderasien. - Gerlands Beitr. Geophysik, 45, S. 381-548, 4 Taf., 13 Abb., Berlin 1935.

Biggs, H. E. J.: Mollusca of the Iranian Plateau. - J. Conchology, 20, S. 342-350, Hertford 1937.

BobEK, H.: Die natürlichen Wälder und Gehölzfluren Irans. - Bonner Geograph. Abh. 8, 60 S., 2 Abb., 4 Taf., 1 Karte, Bonn 1951. - - Beiträge zur klima-ökologischen Gliederung Irans. - Erdkunde, 6, S. 65-84, 6 Abb., 1 Karte, Bonn 1952. - - Klima und Landschaft Irans in vor- und frühgeschichtlicher Zeit. - Geogr. Jber. Österreich, 25, S. 1-42, 4 Abb., 
2 Taf., Wien 1955. - - Forschungen in Persien 1958/59. - Mitt. österr. geograph. Ges, 101, S. 381-388, 2 Taf., Wien 1959 (1959a). - - The Great Kawir of Central Iran. A contribution to the knowledge of its features and formation. - Arid Zone Research Centre Univ. Tehran, 2, 63 S., 17 Taf., Teheran 1959 (1959b).

Boetrger, O.: Die Binnenmollusken Transkaspiens und Chorassans. - Zool. Jb., Systematik, 4, S. 925-982, Taf. 26, 27, Jena 1889.

Braidwood, R. J. \& L.: The Earliest Village Communities of Southwestern Asia. - Cahiers d'histoire mondiale, 1, S. 279-310, Paris 1953.

BüDEL, J.: Bericht über klima-morphologische und Eiszeit-Forschung in Nieder-Afrika. - Erdkunde, 6, S. 104-132, 4 Abb., Bonn 1952.

Butzer, K. W.: Quaternary Stratigraphy and Climate in the Near East. - Bonner Geogr. Abh., 24, 157 S., 4 Taf., 14 Tab., 8 . Abb., Bonn 1958.

Clapp, F. G.: Geology of Eastern Iran. - Bull. Geol. Soc. Amer., 51, S. 1-102, 8 Abb., 13 Taf. Washington 1940.

Coon, C. S.: Cave Explorations in Iran 1949. - Mus. Monogr. Univ. Pennsylvania, 125 S., 15 Taf., 37 Abb., Philadelphia 1951. - - The Excavations at Hotu Cave. - Trans. New York Acad. Sci., ser. 2, 14 (4), New York 1952.

DanIEL, R.: Les gisements préhistoriques de la forêt de Montmorency (Seine- et- Oise). - Bull. Soc. Préhistorique Franc., 51, S. 554-559, 3 Abb., Paris 1954.

Dapples, E. C.: Surficial Deposits of the Deserts of Syria, Trans-Jordan, Iraq and Western Iran. - J. Sedimentary Petro'ogy, 11, S. 124-141, 2 Abb., Menasha 1941.

EвтенaJ, G. H.: A Guide to Iran. -- 4. Aufl., 318 S., 13 Taf., Teheran 1956.

Egeran, N. \& Lahn, E.: Türkiye Jeolojisi - 206 S., 3 Taf., Ankara 1948.

Ehrmann, P.: Weichtiere, Mollusca. - In: Brohmer, Ehrmann, Ulmer „Die Tierwelt Mitteleuropas", 2, 264 S., 147 Abb., 13 Taf., Leipzig (Quelle \& Meyer) 1956.

Favre, J.: Les Mollusques post-glaciaires et actuels du Bassin de Genève. - Mém. Soc. Physique et Histoire naturelle Genéve, 40 (3), S. 171-434, 38 Abb., 14 Taf., Genf 1927.

ForCart, L.: Die Mollusken der nordpersischen Provinz Masenderan und ihre tiergeographische Bedeutung. - Arch. Naturgeschichte, 4, S .404-447, 12 Abb., Leipzig 1935. - - Two new subspecies of Jaminia (Euchondrus) continens (Rosen) from Iran. - J. Conchology, 24, S. 315-317, 3 Abb., Hertfort 1959.

Furon, R.: Manuel de Préhistoire Généra!e. - 484 S., 161 Abb., 8 Taf., 2 Tab., Paris (Рayot) 1958.

Gabriel, A.: Die Lut und ihre Wege. Ergebnisse von drei Iranreisen. - Z. Erdkunde, 10, S. 423 bis 442, 3 Abb., Frankfurt/M. 1942. - -Die Erforschung Persiens. - 359 S., 3 Abb., 24 Taf., Wien (Holzhausens Nfg.) 1952. - - Ein Beitrag zur Gliederung und Landschaftskunde des innerpersischen Wüistengürtels - Festschr. 100-Jahrfeier geograph. Ges. Wien, S. 265-298, 1 Abb., 5 Taf., Wien 1957 (1957a). - - Zur Oberflächengestaltung der Pfannen in den Trockenräumen Zentralpersiens. - Mitt. geograph. Ges. Wien, 99, S. 146160, 3 Taf., Wien 1957 (1957b).

GanjI, M. H.: Iranian Rainfall Data - Arid Zone Research Centre Univ. Teheran, 3, 222 S., 1 Taf., Teheran 1960.

Garrod, D. A. E.: A new Mesolithic Industry: The Natufian of Palestine. - J. Anthropolog. Inst. Great Britain and Ireland, 62, S. 257-269, 5 Abb., 6 Taf., London 1932.

Ghirshan, R.: Fouilles de Sialk près de Kashan 1933, 1934, 1937. - Mus. du Louvre - Dep. Antiquités orientales, Ser. Archéo'ogique, 4, 152 S., 97 Taf., 11 Abb., Paris 1938. - - Iran from the Earliest Times to the Islamic Conquest. - 368 S., 108 Abb., 48 Taf., Penguin Books A 239, Harmondsworth 1954.

Gross, H.: Die Fortschritte der Radiokarbon-Methode 1952-1956. - Eiszeitalter und Gegenwart, 8, S. 141-180, 3 Abb., Ohringen 1957.

Hedin, S.: Zu Land nach Indien durch Persien, Seistan, Belutschistan. - 801 S., 390 Abb., 2 Karten, Leipzig 1910.

Huckriede, R., Kürsten, M. \& Venzlaff. H.: Zur Geologie des Gebietes zwischen Kerman und Sagand (Iran). - Beiheft 51 zum Geol. Jahrb., Hannover 1961 (In Vorbereitung).

Huntington, E.: The Basin of Eastern Persia and Sistan. - Carnegie Inst. Washington, Publ. 26, S. 219-317, Abb. 149-174, Taf. 5-6, Washington 1905. - - Some characteristics of the glacial period in nonglaciated regions. - Bull. Geo!. Soc. Amer., 18, S. 351-388, Taf. 31 bis 39, New York 1907.

Issec, A.: Catalogo dei molluschi raccolti dalla missione italiana in Persia aggiuntavi la descrizione delle specie nuove o poco note. - Mem. Accad. sci. Torino, Ser. 2, 23, S. 387-439, 3 Taf., Turin 1866.

KILIAs, R.: Melania tuberculata (MüLL.) - die malayische Turmdeckelschnecke. - Aquarien und Terrarien, 3 (6), S. 170-171, 2 Abb., Leipzig/Jena 1956. 
Klie, W.: Ostracoda. - In Grimpe, Wagler, Remane: Die Tierwalt der Nord- und Ostsee, 56 S., 51 Abb., 1929. - - Krebstiere oder Crustacea III. Ostracoda, Muschelkrebse. - In DahL, F.: Die Tierwelt Deutschlands und der angrenzenden Meeresteile, 34, 230 S., 786 Abb., Jena 1938.

Linehan, E. J.: Old-New Iran, Next Door to Russia. - National Geographic, 119 (1), S. 44-85, 36 Abb., Washington 1961.

Ministry of Roads. Iranian Meterological Department: Meteoro'ogical Yearbooks 1956, 1957 and Monthly Weather Summary for Synoptic Stations für 1958, 1959, 1960, Teheran.

Misonne, X.: Analyse zoogéographique des Mammifères de l'Iran. - Inst. Sci. naturelles de Belgique, Mém., 2. sér., fasc. 59, 157 S., 24 Abb., 13 Taf., Brüssel 1959.

Movius, H. L.: Comments on Bisitun Cave Material, Iran. - In Coon: Cave Exp'orations in Iran 1949, S. 91, 92, Philadelphia 1951.

MülleR, G. W.: Ostracoda. - In Schulze, F. E.: Das Tierreich, 31, 434 S., 92 Abb., Berlin 1912.

Neuville, R.: Le Préhistorique de Palestine. - Rev. Biblique, 43, S. 237-259, 2 Abb., Taf. 11-19, Paris 1934.

Niedermayer, O. v: Die Binnenbecken des Iranischen Hochlandes. - Mitt. geograph. Ges. München, 14, S. 9-64, 5 Taf., München 1920.

Pilsbry, H. A. \& Bequaert, J.: The aquatic mollusks of the Belgian Congo, with a geographical and ecological account of Congo malacology. - Bull. Amer. Mus. natural History New York, 53, S. 1-659, 77 Taf., 93 Abb., 15 Karten, New York 1927.

Radde, G.: Wissenschaftliche Ergebnisse der im Jahre 1886 Allerhöchst befohlenen Expedition nach Transkaspien und Nord-Chorassan. - Petermanns Mitt., Ergänzungsheft 126, 195 S., 1 Karte, Gotha 1898.

Rechinger, K. H.: Vegetationsbilder aus dem nördlichen Iran. - Vegetationsbilder, Reihe 25, Heft 5, 12 S., 1 Abb., Taf. 25-30, Jena 1939. - - Grundzüge der Pflanzenverbreitung im Iran. - Verh. Zoo'og.-Botan. Ges. Wien, 92, S. 181-188, Wien 1951.

Remane, A. \& Schlieper, C.: Die Biologie des Brackwassers. - Die Binnengewässer, 22, 348 S., 139 Abb., 43 Tab., Stuttgar 1958.

Reuter, A.: Der Pfortner Berg bei Gera und seine mesolithischen Werkzeuge. - Alt-Thüringen, 1, S. 43-51, 7 Abb., Weimar 1955.

Roger, J.: Mollusques Fossiles et Subfossiles du Bassin du Lac Rodolph. - Mission Scientifique de l'Omo 1932-1933, 1, Géologie - Anthropologie, fasc. 2, S. 119-155, 2 Abb., 2 Taf., Paris 1943.

Scharlau, K.: Zum Problem der Pluvialzeiten in Nordost-Iran. - Z. Geomorphologie, N. F. 2 (2), S. 258-277, 6 Abb., Berlin 1958.

Schlesch, H.: Kleine Mitteilungen X. - Arch. Molluskenkunde, 66, S. 29-49, Taf. 2-6, Frankfurt 1934.

Sedlacek, A. M.: Sande und Gesteine aus der Südlichen Lut und Persisch-Belutschistan. - S.-B. Ósterr. Akad. Wiss., math.-naturw. Kl., Abt. 1, 164, S. 607-658, 7 Abb., Wien 1955.

Starmühlner, F. \& Edi.Auer, Ae.: Ergebnisse der Österreichischen Iran-Expedition 1949/50. Beiträge zur Kenntnis der Molluskenfauna des Iran. - S.-B. Ósterr. Akad. Wiss., math.naturw. Kl., Abt. 1, 166, S. 435-494, 17 Abb., 3 Taf., Wien 1957.

Stratil-Sauer, G.: Die p'eistozänen Ablagerungen im Innern der Wüste Lut. - Festschr. 100-Jahrfeier Geograph. Ges. Wien, S. 460-484, 3 Abb., W/ien 1957.

Turvil Le-Petre, F.: Excavations in the Mugharet El-Kebarah. - J. Anthropol. Inst. Great Britain and Ireland, 62, S. 271-276, 3 Abb., 3 Taf., London 1932.

Vanden Berghe, L.: De Stand van de archaeologische onderzoekingen in Iran. - Ex Oriente Lux, Jaarbericht 13, S. 347-393, Abb. 36-40, 14 Taf., Leiden 1954.

Vaufrey, R.: Notes sur le Capsien. - L'Anthropologie, 43, S. 457-483, 20 Abb., Paris 1933.

Vavra, V.: Ostracoda, Muschelkrebse. - In Brauer: Die Süßwasserfauna Deutschlands, 11, S. 85 bis 119, Abb. 346-484, Jena 1909.

Walther, J.: Das Gesetz der Wüstenbildung. - 175 S., 50 Abb., Berlin (Rermer) 1900.

WENZ, W.: Land- und Süßwassermollusken aus fluvioäolischen Ab'agerungen von Hadramaut (Südarabien). - Arch. Molluskenkunde, 75, S. 148-151, 240-241, 3 Abb., Frankfurt/M. 1943.

Wenzel, H.: Forschungen in Inneranatolien I. Aufbau und Formen der Lykaonischen Steppe. Schr. geograph. Inst. Univ. Kiel, 5 (1), 48 S., 14 Abb., 3 Anlagen, Kiel 1935.

Wheeler, M.: Early India and Pakistan to Ashoka. - 241 S., 32 Abb., 40 Taf., London (Thames \& Hudson) 1959.

Manuskr. eingeg. 27. 5. 1961.

Anschrift des Verf.: Dr. R. Huckrıede, Bundesanstalt für Bodenforschung, Hannover, Wiesenstr. 1. 